\section{Wietig \\ S. Williams \\ M. Davids \\ M. Kerscher}

\title{
Kulturgeschichtliche Aspekte heller Haut
}

\section{Historic Aspects of Fair Skin}

\section{Zusammenfassung}

Das Merkmal „helle Haut“ war in der Entwicklungsgeschichte des westlichen Zivilisationsprozesses nie ein wertfreies Merkmal des Menschen, sondern nahm kulturgeschichtlich oft eine Sonderstellung ein. Der Ursprung dieser Sonderstellung geht auf die antiken Ägypter zurück, bei denen das Fleisch der Götter metaphysisch mit dem hellen Licht der Sonne gleichgesetzt wurde, und auf die griechische Liebesgöttin Aphrodite, die als hellhäutige, blonde Frau dargestellt wurde. Helle Haut, der über lange Epochen mit bleihaltigen, langfristig hautschädigenden Abdeckpasten nachgeholfen wurde, wies später auf eine Zugehörigkeit zur Oberschicht hin, da sie das nicht Angewiesensein auf Arbeit im Freien visualisierte.

Erst im „Jetset“-Zeitalter des endenden 20. Jahrhunderts löste die prestigebesetzte Bräune das „Ideal“ der hellen Haut ab. Sonnengebräunte Haut visualisierte materielle Unabhängigkeit mit der Möglichkeit zu reisen, während helle Haut mit Blässe und Krankheit assoziiert wurde. Das Image der sonnengebräunten Haut wurde zwar wenige Jahre später durch Hautkrebs- und Antiaging-Kampagnen relativiert, jedoch nicht vollständig umgekehrt. Auch heute noch gilt ein leicht gebräunter Teint als erstrebenswert, wenn auch nicht um jeden Preis.

\section{Abstract}

The bodily feature of „fair“ skin has never been a neutral phenotypic feature in western societies, but has always taken a special position. The origin of this goes back to ancient Egypt, where the flesh of the gods was metaphysically equated to the bright light of the sun and the Greek love goddess Aphrodite was depicted as a fair-skinned, blond woman. Later in history fair skin indicated the membership of the higher social classes, as "normal" people had to work outdoors, therefore quickly acquiring a tan. For long time fair skin was enhanced by lead-containing camouflagepastes, which caused severe damage to the skin after long-term application.

The "ideal" of a fair teint, which had remained constant over many centuries, was replaced by the prestigious sun-tan in the jet-set age of the late $20^{\text {th }}$ century. Well-tanned skin then came to represent material independence and ability to travel, while fair skin was seen as somewhat unhealthy. This image has changed to some extent through educational skin cancer and antiaging campaigns which stress the harmful effects of ultraviolet irradiation. Tanned skin however is still seen as desirable, but no longer at any cost.
„Helle Haut“ war seit Beginn der Entwicklungsgeschichte des westlichen Zivilisationsprozesses kein wertfreies Körpermerkmal wie viele andere phänotypische Merkmale des Menschen, sondern nahm bezüglich kulturgeschichtlicher Aspekte oft eine Sonderstellung ein. So blieb die helle Haut im immerwährenden
Wandel der Schönheitsideale durch alle Stilepochen bis ins 20. Jahrhundert ein unverändertes Statussymbol. Erst im „Jetset“-Zeitalter des endenden 20. Jahrhunderts löste die prestigebesetzte Bräune das „Ideal“ der hellen Haut ab und helle Haut wurde oft mit Blässe und Krankheit assoziiert. Das Image (von 
lat. Imago, Bild) der sonnengebräunten Haut wurde jedoch bereits wenige Jahrzehnte später durch Hautkrebs- und Antiaging-Kampagnen des Mythos Alterslosigkeit teilweise wieder revidiert, wobei trotz intensiver Aufklärungsmaßnahmen noch heute eine „gesunde Bräune“ bei vielen Menschen als erstrebenswert gilt.

Der Ursprung des „Ideals“ der hellen Haut, das über die lange Jahrhunderte konstant bleiben sollte, geht auf die antiken Ägypter zurück, die sich gleichermaßen um die Schönerhaltung der Lebenden wie auch die ewige Schönheit der Toten bemühten. Die täglichen Reinigungs- und Schminkprozeduren standen in engem Zusammenhang mit den von Priesterärzten ausgeführten Mumifizierungstechniken, die die sterbliche Hülle vor dem Zerfall bewahren sollten. Bei den antiken Ägyptern wurde das Fleisch der unsterblichen Götter durch Gold symbolisiert, das metaphysisch mit Licht und der Urenergie der Sonne gleichgesetzt wurde. Dieser strahlende, alles übertreffende Lichtglanz der Göttlichkeit prägte die spätere kulturelle Präferenz für helle Haut. Außergewöhnliche natürliche Schönheit wurde darum seit jeher als Gottesgeschenk angesehen und die kosmetisch-technische Annäherung an das „göttliche Ideal“ wurde in den hierarchischen Klassengesellschaften nur gesellschaftlich herausragenden Persönlichkeiten zugestanden. Die helle Haut, die „vornehme Blässe“, visualisierte auch körperästhetisch die Zugehörigkeit zur Oberschicht, weil eine mögliche Bräunung durch den Zwang zur Arbeit im Freien vermieden werden konnte. Über die Hautfarbe wurden die historischen Machtstrukturen und die damit verbunden Besitzverhältnisse angezeigt. Weiß wurde somit zur Farbe der hegemonialen Kultur.

Helle Haut war jedoch nicht nur Merkmal für die Zugehörigkeit zu einer höheren Schicht, sondern symbolisierte auch Jugend und Unberührtheit. Da die Haut bestimmter Lokalisationen in der Schwangerschaft nachdunkeln kann (zum Beispiel Linea nigra), versinnbildlichte helle Haut die Jungfräulichkeit und machte besonders begehrenswert. In der Kunst wird helle Haut geschlechtsspezifisch zwischen Männern und Frauen nuanciert unterschiedlich dargestellt. So wurde das Inkarnat, der Fleischton der Körperhaut, von Frauen immer etwas heller als das der Männer gewählt.

Das „Ideal“ der hellen Haut war jedoch stets mit dem Streben nach reiner, makelloser Haut ohne sichtbare Läsionen und Alterserscheinungen verbunden. Im Papyros Ebers, einer ägyptischen medizinischen Textsammlung aus dem Jahre 1552 v. Chr., berühren folgende Kapitel der Rezepturvorschriften zur Wiedererlangung der Gesundheit die Hautverschönerung und deren Jungerhaltung (Abb.1):

„87, 3-4. Desgl. um die Hautfarbe zu ändern, 6-8. Desgl. zur Vertreibung der Gesichtspickel, 8-15. Desgl. um dem Gesichte ein glattes Ansehen zu geben, 15 - 17. Desgl. um die Falten im Gesicht zu vertreiben." [1]

Bei den antiken Griechen wird das Bemühen um Schönheit unter dem Begriff Kalokagathia ausgedrückt, der als altgriechisches Bildungsziel Schön- wie auch Gutsein umfasst und dem ästhetischen sowie ethischen Ideal der Vollkommenheit entsprach. Der Philosoph Demokritos (um 460 - 371 v. Chr.) und der Schriftstel-

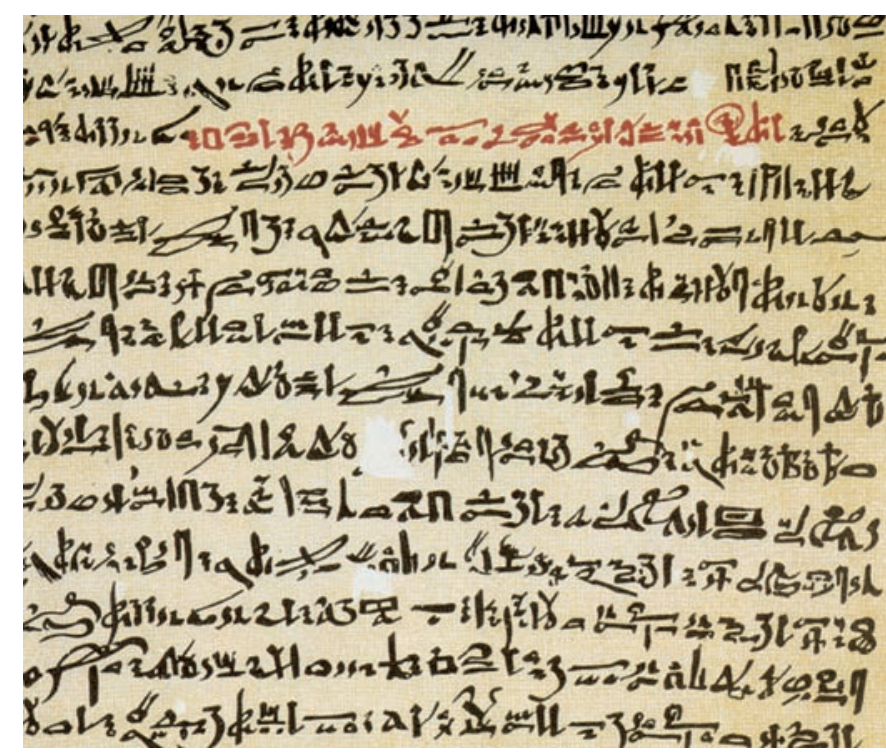

Abb. 1 Beispiel hieratischer Schrift. Detail des Papyros Ebers (1552 v. Chr.); aus: Lohse-Jasper R. Die Farben der Schönheit, Eine Kulturgeschichte der Schminkkunst. Hildesheim: Gerstenberg Verlag, 2000:19.

ler Athenaios (um 200 n. Chr.) empfahlen, die Haut nach der Reinigung mit Fetten zu salben, um deren Glätte zu erhalten [2]. Bereits die antiken Griechinnen verwendeten Bleiweiß, um ihren Teint aufzuhellen.

Aphrodite, die griechische Göttin der Liebe und Schönheit, die spätere römische Venus, entstammt den göttlichen Gefilden des Olymp. Ihre Anmut, ihre helle Haut, ihr blondes Haar und ihre „beglückende Lichtfülle“ stellten für die alten Griechen wichtige Merkmale ihrer göttlichen Ausstrahlung dar. Der auf der Erde am hellsten erscheinende Planet, der Morgen- und Abendstern, wurde als Venus, der Stern der Liebesgöttin, bezeichnet. Er erlangte bereits in der babylonischen Astralmythologie als positives Sinnbild der Ischtar hohe Bedeutung. Weil dieser Archetyp der Liebesgöttin auch in den folgenden Jahrhunderten Schönheit, Jugendlichkeit und Attraktivität symbolisierte, eiferten ihm Frauen aller Epochen nach (Abb. 2).

Der griechische Begriff Kosmetik (griechisch für Haut- und Schönheitspflege) steht etymologisch in Verbindung mit dem Kosmos (griechisch für Ordnung), der Weltordnung und mit dem Weltall, wobei der Mensch metaphysisch als Mikrokosmos (griechisch für die kleine Welt) im Makrokosmos (griechisch für das Weltall) verstanden wird. Kosmetik betreiben bedeutet also übertragen, sich in Resonanz und Einklang mit der Harmonie des Universums und des Göttlichen zu bringen. Das „Ideal“ heller Haut stand in der Antike nicht nur in Abhängigkeit ihrer Oberflächenbeschaffenheit, sondern auch ihres räumlichen Bezugs. Beispielsweise entsprechen die Proportionen des Goldenen Schnitts den Zahlenverhältnissen der Umlaufbahn der Venus zur Sonne und zur Erde. Auch das seit der Antike den Menschen symbolisierende Pentagramm, das Fünfeck, bildet sich durch die Positionen der unteren Konjunktion von Sonne und Venus im Tierkreis [3]. Die Proportion des Menschen, die anthropometrische Verhältnismäßigkeit, bestimmte daher seit der Antike das mikrokosmische Schönheitsideal des Menschen in Maß und Zahl. 


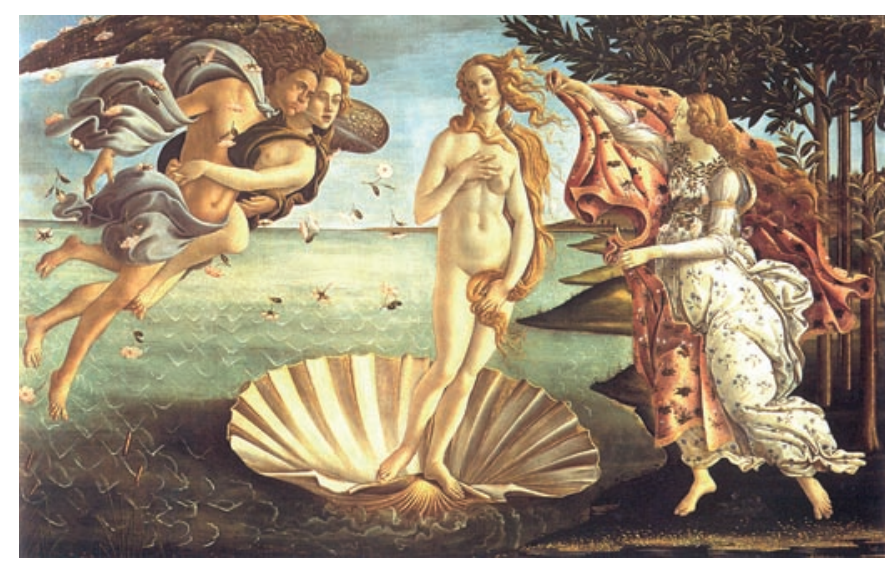

Abb. 2 Sandro Botticelli (1445-1510) „Die Geburt der Venus“, Uffizien, Florenz, 1486; aus: Toman R (Hrsg). Die Kunst der italienischen Renaissance, Architektur, Skulptur, Malerei, Zeichnung. Köln: Könemann, 1994:283.

Durch die philosophische Urbild-Abbild-Theorie der Antike, nach der eine schöne Seele in einem schönen Körper wohne, wurden Schönsein, Gutsein und Gesundheit gleichgesetzt. In der physiognomischen und literarischen Tradition wurden häufig Analogien zwischen Helligkeit und Transparenz der Haut sowie Empfindsamkeit und einem positiven Durchscheinen der Emotionen gezogen. Die Hautpigmentierung wurde daher oft nicht allein nach „ästhetischen“, sondern auch nach moralischen Werthierarchien klassifiziert. Die Haut wurde sinnbildlich als Spiegel der Seele und des körperlichen Wohlbefindens angesehen. Heller Glanz der Haut sollte von unversehrter Oberfläche und damit Gesundheit zeugen. Diese Emanation ließ helle Haut erstrebenswerter und attraktiver erscheinen.

Dem „Ideal“ standesabgrenzender schneeweißer Haut wurde über lange Epochen bis hin zur Renaissance mit abdeckenden, weißen Kosmetika nachgeholfen. Der römische Dichter Ovid (Publius Ovidius Naso, 43. v. Chr. bis etwa $17 \mathrm{n}$. Chr.) beschreibt in einem erhaltenen Fragment über Kosmetik „Medicamina faciei femineae" eine Gesichtsmaske für die Schönerhaltung heller Haut:

„Dic age, cum teneros somnus dimiserit artus, candida quo possint ora nitere modo....

sextantemque trahat cummi cum semine Tusco;

huc nouies tanto plus tibi mellis eat:

quaecumque afficiet tali medicamine uultum,

fulgebit speculo leuior illa suo." [4]

„Lernt jetzt, wie das Gesicht, wenn der Schlaf euch befreit die zarten Glieder, sich glänzend schmücke mit strahlendem Weiß. ...Thu zwei Unzen dazu von Tustischen Körnern und Gummi, und neunmal so viel Honig noch gieße darein. Wenn du dir dann das Gesicht einreibst mit der obigen Mischung, wird es glänzend, dass selbst heller dein Spiegel nicht strahlt.“ [5]

Eine berühmte, verschwenderische Anwenderin von Bleiweiß in Zeiten der Renaissance war die englische Königin Elisabeth I. (1558 - 1603), die daher auch als „Elfenbein-Regentin“ in die Geschichte einging. Die Langzeitanwendung Blei-haltiger kosmeti- scher Zubereitungen führte jedoch zu einer deutlich sichtbaren Hautschädigung. Im Alter ließ Königin Elisabeth I. daher alle Spiegel in ihrem Palast entfernen, um den Verfall ihres Gesichtes nicht länger mit ansehen zu müssen [6].

Im kulturell wegweisenden Frankreich der ersten Hälfte des 17. Jahrhunderts galt die künstliche Körpermodifikation als Verfeinerung des Naturzustandes, die auch einer Bewahrung der standesabgrenzenden Distinktion diente. Bleiweißschminken, Perücken, Puder und Parfums der „trockenen Toilette“ waren daher prestigeverpflichtend. Auch Altersunterschiede zwischen Kindern und Greisen wurden symbolisch durch Bleiweißschminke aufgehoben und suggerierten Zeitlosigkeit und Unantastbarkeit. Die exzessiven Schminktechniken blieben jedoch auch unter Zeitgenossen nicht ohne satirische Kommentare, wie die Kritik des Predigers und Volksschriftstellers Abraham a. S. Clara (1644-1709), eigentlich Johann Ulrich Megerle, am Schminken der Damen belegt:

„1) Abrahamisches Gehab dich wohl ...: ihre Falten überschmiert sie mit Curnisoll, Bleyweiß und anderer Schminke, hat beynebens etliche helffenbeinerne Zähn im Maul, welche ihr der Artzt eingesetzet.“ [7]

Im Zeitalter der Aufklärung wird in Ratgebern wie „Anmuth und Schönheit aus den Misterien der Natur und Kunst für ledige und verheirathete Frauenzimmer mit Kupfern, Berlin 1797“ vor den gesundheitsschädlichen Schminken gewarnt:

„Schädliche Schönheitsmittel.

Es gibt bekanntlich rothe und weiße Schminke. Die gewöhnlichsten weißfärbenden Mittel sind: Der Sublimat, weißer Vitriol, Perlen, Benzoe, Wismuth, Bleiweiß, und hiervon vorzüglich das Kremserweis, Koboldpräcipitat, Alabaster und weißer Puder. Roth färbt Karmin, Zinnober, Kugellack, die mit Zinnober gemachte Seife, Talch (ein venetianischer kalkartiger Stein) mit Saflor gefärbt, und die Blume der Amaranthe. Brandwein macht auch auf eine kurze Zeit die Haut roth, wegen seiner erwärmenden und zusammenziehenden Kräfte.“ [8]

Erst im Blick anderer nimmt der eigene Körper Kontur einer Ethnie an. Wir sind gewohnt, Zeichen des Körpers geografisch, national und kulturell zu deuten. Durch die binären Denkstrukturen des christlichen Abendlandes wurden physische Merkmale wie Konstitution und Hautfarbe in der Ethnologie und Anthropologie oft dual mit positiven und negativen Eigenschaften besetzt. Dies geschieht am folgenschwersten in rassentheoretischen Diskursen. Für den Rassentheoretiker Arthur de Gobineau (1816 - 1882) zum Beispiel war die Hautfarbe ein zentrales Kriterium [9].

Weil dunkle Haut sich weniger sichtbar verändert, galt sie bei einigen Menschen als undurchdringlicher und verhüllend (englisch für hide). Auch die Etymologie von Color verweist auf celare (lat. für verstecken). Danach haben Farbpigmente zugleich bergenden bzw. verbergenden Charakter. In den USA steht Color synonym für Race. Durch die in vielen Ländern wie auch den USA gängige Unterscheidung in white und colored (als degradierende Subsummierung aller „nicht-weißen“ Menschen) wird die anthropologische und ideologische Frage von Farbigkeit und Farblo- 
sigkeit aufgeworfen, die die binäre Struktur des Denkens mit den Konstrukten von Farbig und Weiß der folgenschweren „Epidermical hierarchy" begründet.

Jedoch gibt es auch gegenüber bestimmten Aspekten heller Haut Vorbehalte. So wird seit Beginn des Jetset-Zeitalters helle Haut oftmals mit Blässe und Krankheit assoziiert, während sonnengebräunte Haut als „gesunde Hautfarbe“ bezeichnet wird. Ausgeprägte Vorbehalte zeigten sich von jeher gegenüber der Pigmentlosigkeit des Albinismus. In der Literatur wird die Perzeption pigmentfreier Haut von Franz Kafka (1883-1924) und den zeitgenössischen Schriftstellern Plath und John Edgar Wideman als mangelhafte, formlose, kranke und leblose Hülle vorgestellt. Wideman beschreibt in seinem Roman "Sent for You Yesterday“ (1983) den Albino Brother Tate als "dead in bag of white skin“ [10]. Menschen mit Albinismus wurden auch in vielen Religionen als „un-menschlich“ angesehen, für die normale soziale Regeln nicht galten. Darum begegnete man ihnen mit Scham, Tötung oder - weil man glaubte, sie seien auferstandene Tote mit Apotheose, die sie schützte. Erst im Zeitalter der Aufklärung wird nach dem Werk Georges Louis Leclerc Buffons (1707-1788) „Histoire naturelle“ (1777) der Albinismus als eine individuelle Variante innerhalb der Spezies entmystifiziert.

Das „ideale“ Körperbild, ursprünglich den Göttern und höheren Ständen vorbehalten, wird seit der Antike bis heute als Statussymbol der sozialen Macht angestrebt. Das über Jahrhunderte fest verankerte „Ideal“ der hellen Haut wurde Ende des 20. Jahrhunderts aufgeweicht, unter anderem durch das Reisen in ferne Länder als Symbol für materielle Unabhängigkeit. Trotz Eiferns nach sonnengebräunter Haut blieb jedoch in den westlichen Gesellschaften weiterhin das „Idealbild“ des hellen Hautphänotyps bestehen. Das Streben nach stark sonnengebräunter Haut durch intensives Sonnenbaden, das vor 10 bis 30 Jahren seinen Höhepunkt hatte, wurde durch Aufklärungskampagnen über die schädlichen Wirkungen des ultravioletten Lichtes relativiert, jedoch nicht vollständig aufgehoben. Auch heute noch gilt ein leicht gebräunter Teint als erstrebenswert, wenn auch nicht um jeden Preis. Davon zeugt zum Beispiel der derzeit zu beobachtende Boom Dihydroaceton-haltiger „Selbstbräuner“.

\section{Literatur}

${ }^{1}$ Papyros Ebers. Das hermetische Buch über die Arzneimittel der Alten Ägypter in hieratischer Schrift, Leipzig 1875. Osnabrück: Biblio Verlag, 1987

2 Sigismund R. Die Aromata in ihrer Bedeutung für Religion, Sitten, Gebräuche, Handel und Geographie des Alterthums bis zu den ersten Jahrhunderten unserer Zeitrechnung. Leipzig: C. F. Winter'sche Verlagshandlung, 1884

${ }^{3}$ Ströter-Bender J. Liebesgöttinnen, Von der Großen Mutter zum Hollywoodstar. Köln: Dumont, 1994

${ }^{4}$ Ovidi Nasonis P. Amores, Medicamina faciei femineae, Ars amatoria, Remedia Amoris, Editit, Brevique Adnotatione Critica. Kenney EJ (Hrsg.). Oxford: Oxford University Press, 1989

${ }^{5}$ Rimmel E. Das Buch des Parfums, Die klassische Geschichte des Parfums und der Toilette. Dreieich: Hesse \& Becker, 1985

${ }^{6}$ Liggett J. Die Tyrannei der Schönheit. München: Wilhelm Heyne, 1989

${ }^{7}$ Schultz A. Alltagsleben einer deutschen Frau zu Anfang des achtzehnten Jahrhunderts. Leipzig: Hirzel, 1890

${ }^{8}$ Anmuth und Schönheit aus den Misterien der Natur und Kunst für ledige und verheirathete Frauenzimmer mit Kupfern, Berlin, 1797. Reprint der bibliophilen Taschenbücher. Dortmund: Harenberg Kommunikation, 1978

${ }^{9}$ Gernig K. Fremde Körper im Visier. In: Randow G (Hrsg.). Wie viel Körper braucht der Mensch. Standpunkte zur Debatte für den Deutschen Studienpreis. Hamburg: Edition Körber-Stiftung, 2001: 119-128

${ }^{10}$ Benthien C. Haut, Literaturgeschichte - Körperbilder - Grenzdiskurse.

2. Aufl. Hamburg: Rowohlts Enzyklopädie, 2001 